\title{
Sequentially Bornological Compact Space
}

\author{
Fatma Kamil Al-Basri \\ Department of Mathematics, College of Education, University of Al-Qadisiyah, Iraq. \\ Email: Fatma.AlBasri@qu.edu.iq
}

Received:- $\quad 16 / 5 / 2017$

Accepted:-24/10/2017

\begin{abstract}
:
In this paper, we introduce the definitions of sequentially bornological continuous mapping and sequentially bornological compact spaces in bornological vector space. We investigate the properties of these concepts. Also, we give the relation between sequentially bornological compact spaces and complete bornological vector spaces.
\end{abstract}

Keywords: bornological vector space, sequentially bornological compact spaces, bornological convergence, sequentially bornological continuous map.

Physical Classification QA299.6- 433 


\section{Introduction:-}

In(1971), H.Hogbe-Nlend introduced the concepts of bornology on a set [1]. Many workers such as Dierolf and Domanski, Jan Haluska and others had studied various bornological properties [2]. G.W. Macky, who was the first to study convergence systematically, since 1942, such a notion of convergence in the particular context of its theory of "linear systems" [3]. Complete bornological vector spaces was studied and investigated in [4]. We define in section two bornological open (closed) map and sequentially bornological continuous map in bornological vector spaces (bvs) and obtain some results. Sequentially bornological compact spaces has been investigated in section three after that we introduces the relation between them and bornological complete space, sequentially bornological compact in product bornological vector spaces and we get the main results which is in concerned to them.

\subsection{Definition [5]:-}

A bornology on a set $X$ is a family $\boldsymbol{\beta}$ of subsets of $X$ satisfying the following axioms:

(i) $\beta$ is a covering of $X$, i.e. $X=\bigcup_{B \in \beta} B$;

(ii) $\boldsymbol{\beta}$ is hereditary under inclusion i.e. if $A \in \boldsymbol{\beta}$ and $\mathrm{B}$ is a subset of $X$ contained in , then $B \in \boldsymbol{\beta}$;

(iii) $\boldsymbol{\beta}$ is stable under finite union.

A pair $(X, \beta)$ consisting of a set $X$ and a bornology $\boldsymbol{\beta}$ on $X$ is a bornological space, the elements of $\beta$ are called the bounded subsets of $X$.

\subsection{Example [1]:-}

If $\mathbb{R}$ be a field of real numbers with the absolute value. The collection:

$\boldsymbol{\beta}=\{A \subseteq \mathbb{R}: A$ is bounded subset of $\mathbb{R}$ in the usual sense for the absolute value $\}$. Then $\beta$ is a bornology on $\mathbb{R}$ called the Canonical Bornology of $\mathbb{R}$.

\subsection{Definition [4] :-}

Let $E$ be a bornological vector space. A sequence $\left\{x_{n}\right\}$ in $E$ is said to be converge bornologically to 0 if there exists a circled bounded subset $B$ of $E$ and a sequence $\left\{\lambda_{n}\right\}$ of scalars tending to 0 ,such that $x_{n} \in B$ and $x_{n} \in \lambda_{n} B$, for every integer $n \in N$. Bornological convergence is also called MackyConvergence writes $x_{n} \stackrel{M}{\rightarrow} 0$ If $\left(x_{n}-x\right) \stackrel{M}{\rightarrow} 0$, and we write $x_{n} \stackrel{M}{\rightarrow} x$.

\section{4 proposition [6]:-}

Let $x_{n} \stackrel{M}{\rightarrow} 0$ if and only if every subsequence of $\left\{x_{n}\right\}$ converges to 0 .

\subsection{Proposition [6]:-}

A sequence $\left\{x_{n}\right\}_{n \in N}$ in a product bornological vector space $\prod_{i \in I} E_{i}$ converges bornologically to $\mathrm{y}$ if and only if The sequence $\left\{x_{n}^{i}\right\}_{i \in I}$ converges bornologically to $y_{i}$ in bornological vector space $E_{i}$.

\subsection{Definition [4]:-}

Let $E$ be a bornological vector space, let $\left\{x_{n}\right\}_{n \in N}$ be a sequence in $E$.We call $\left\{x_{n}\right\}$ a bornological Cauchy sequence or (a MackeyCauchy sequence) in $E$ if there exists a circled bounded subset $S \subseteq E$ and a null sequence of positive scalars $\left\{\lambda_{n}\right\}$ such that $\left\{x_{n}\right\} \in S$ and $x_{n}-x_{m} \in \lambda_{m} S$ for all $n, m \in N$ with $n \geq m$.

\subsection{Theorem [4]:-}

Every Mackey- Cauchy sequence in a bornological vector space $E$ which has a bornologically convergent subsequence, it is bornologically convergent.

\subsection{Definition [1]:-}

Let $E$ be a bornological vector space. A subset $A \subseteq E$ is said to be bornologically open or Mackey open (briefly, b-open or Mopen) if for every sequence $\left\{x_{n}\right\}_{n \in N} \subseteq E$ converging to a point $x \in A$ then $x_{n} \in A \forall n>$ $n_{0}$.

\subsection{Definition [1]:-}

Let $E$ be a bornological vector space. $A$ subset $A \subseteq E$ is said to be bornologically closed or Mackey closed (briefly, b- closed or M- closed) if the following condition satisfy:

$\left\{x_{n}\right\}_{n \in N} \subset A$ and $x_{n} \stackrel{M}{\rightarrow} x$ in $E$ imply that $x \in$ A.

\subsection{Definition [1]:-}

Let $E$ be a bornological vector space. The bornological closure briefly, b-closure or M-closure of a set $A \subseteq E$ denoted $\bar{A}$ is the intersection of all bornologically closed (bclosed) subset of $E$ containing $A$. 


\subsection{Remark :-}

Let $E$ be a bornological vector space then:

i. if $A \subset B \Rightarrow \bar{A} \subset \bar{B}$.

ii. $\bar{A}$ is the smallest b-closed subsets of $E$ containing $A$.

\section{Some Sequentially Bornological Map}

In this section we define bornological open map (closed map) depended on open (closed) set in bornological vector space (bvs) and we define sequentially bornological continuous map and investigate the properties concerning with them.

\subsection{Definition:-}

Let $E$ and $F$ be bornological vector spaces and $f$ be a mapping of $E$ into $F$ then:

i. $\quad f$ is said to be bornological open map or Mackey open map (briefly, b-open or M-open) if $f(A)$ is b-open in $F$ for every $A$ is b-open in $E$.

ii. $\quad f$ is said to be bornological closed map or Mackey closed map (briefly, bclosed map or M-closed map) if $f(B)$ is b-closed in $F$ for every $B$ is b-closed in $E$.

\subsection{Theorem:-}

Let $E$ and $F$ be two bornological vector spaces. A mapping $f: E \rightarrow F$ is b-closed if and only if $\overline{f(A)} \subset f(\bar{A})$ for every $A \subset E$.

Proof: clear

\subsection{Definition:-}

Let $E, F$ be a bornological spaces and let $f$ be a mapping from $E$ into $F$. We say that $f$ is a sequentially bornological continuous at a point $x$ if for any sequence $\left\{x_{n}\right\}_{n \in N}$ in $E$, $x_{n} \stackrel{M}{\rightarrow} x$ then $f\left(x_{n}\right) \rightarrow f(x)$ in $F$ If $f$ is a sequentially bornological continuous at every point $x$ in $E$, then $f$ is called sequentially bornological continuous.

2.4 Example:- let $(\mathbb{R}, \boldsymbol{\beta})$ be the canonical bornological vector space over $\mathbb{R}$ and $f:(\mathbb{R}, ß) \rightarrow(\mathbb{R}, ß)$ defined $f(x)=x$ then $f$ is a sequentially bornological continuous.

\subsection{Theorem:-}

Let $f: E \rightarrow F \quad$ and $\quad g: E \rightarrow F \quad$ be sequentially bornological continuous then:

i. $\quad c f$ sequentially bornological continuous $\forall c \in K$.

ii. $f+g$ sequentially bornological continuous.

iii. $f . g$ is a sequentially bornological continuous.

Proof

1. Let $x \in E$ and $\left\{x_{n}\right\}$ be a sequence in (bvs) $E$, such that $x_{n} \stackrel{M}{\rightarrow} x$. Since $f$ is a sequentially bornological continuous at

$x$, then $f\left(x_{n}\right) \rightarrow f(x)$ in $E$ implies $c f\left(x_{n}\right) \rightarrow c f(x), \quad \forall c \in K$.then for all $x$ we have cf sequentially bornological continuous.

2. Let $x \in E$ and $\left\{x_{n}\right\}$ be a sequence in (bvs) $E$, such that $x_{n} \stackrel{M}{\rightarrow} x$. Since $f, g$ are sequentially bornological continuous at $x$ then $f\left(x_{n}\right) \rightarrow f(x)$ in $F$ and $g\left(x_{n}\right) \rightarrow g(x) \quad$ in $F$, then $f\left(x_{n}\right)+g\left(x_{n}\right) \rightarrow f(x)+g(x)$ in $F$.we have $(f+g)\left(x_{n}\right) \rightarrow(f+g)(x)$ in $F$, then $f+g$ is a sequentially bornological continuous at every point $x$ in $E$. Then $f$ is sequentially bornological continuous.

3. Let $x \in E$ and $\left\{x_{n}\right\}$ be a sequence in (bvs) $E$, such that $x_{n} \stackrel{M}{\rightarrow} x$. Since $f, g$ are sequentially bornological continuous at $f$ then $f\left(x_{n}\right) \rightarrow f(x)$ in $F \quad$ and $g\left(x_{n}\right) \rightarrow g(x)$ in $F$ then $f\left(x_{n}\right) \cdot g\left(x_{n}\right) \rightarrow f(x) \cdot g(x)$ in $F$, hence $(f \cdot g)\left(x_{n}\right) \rightarrow(f \cdot g)(x)$ in $F$ at every $x$ in $E$ then $f \cdot g$ are sequentially bornological continuous.

\subsection{Theorem:-}

Let $E$ and $F$ be a bornological vector space and $A$ a non-empty subset of $E$ if $f: E \rightarrow F$ is a sequentially bornological continuous then the restriction $f_{A}$ is a sequentially bornological continuous where $A$ has the relative bornology $B_{A}$.

\section{Proof}

Let $x \in A$ and $\left\{x_{n}\right\}_{n \in N} \subseteq A$ such that $x_{n} \stackrel{M}{\rightarrow} x$ in $A$

Since $A \subseteq E$ then $x_{n} \stackrel{M}{\rightarrow} x$ in $\mathrm{E}$ 
Since $f: E \rightarrow F$ is a sequentially bornological continuous then if $x_{n} \stackrel{M}{\rightarrow} x$ we have $f\left(x_{n}\right) \rightarrow f(x)$ hence $f_{A}$ is a sequentially bornological continuous function.

\subsection{Theorem:-}

A mapping f from a (bvs) $E$ into (bvs) $F$ is sequentially bornological continuous, for every $B$ is closed set in $F$ then $f^{-1}(B)$ is closed set in $E$.

\section{Proof}

Let $B$ is b-closed set in $F$, if $f^{-1}(B)=$ $\phi$ and thus the proof is complete .If $f^{-1}(B) \neq$ $\phi$. Let $x_{n} \in f^{-1}(B)$ such that $x_{n} \stackrel{M}{\rightarrow} x$ in $f^{-1}(B) \in E$, we have $f\left(x_{n}\right) \in B$. Since $f$ is sequentially bornological continuous then $f\left(x_{n}\right) \rightarrow f(x)$. Since B is b-closed set then $f(x) \in B$. We have $x \in f^{-1}(B)$ then $f^{-1}(B)$ is b-closed set in $E$.

\subsection{Theorem:-}

Let $E$ and $F$ be a (bvs), if $f: E \rightarrow F$ is sequentially bornological continuous then:

$$
\begin{aligned}
& \text { i. } f(\bar{A}) \subseteq \overline{f(A)} \text { for every } A \subseteq E \text {. } \\
& \text { ii. } \overline{f^{-1}(B)} \subseteq f^{-1}(\bar{B}) \text { for every } \\
& B \subseteq F \text {. }
\end{aligned}
$$

\section{Proof}

i. Let $f$ be sequentially bornological continuous. Since $\overline{f(A)}$ is b-closed in , $f^{-1}(\overline{f(A)})$ is b-closed set in $E$ (theorem 2.7).

$\overline{f^{-1}(\overline{f(A)})}=f^{-1}(\overline{f(A)})$. Since $f(A) \subseteq \overline{f(A)} \Rightarrow A \subseteq f^{-1}(f(A)) \subseteq$ $f^{-1}(\overline{f(A)}), \quad$ then $\quad \bar{A} \subseteq f^{-1}(\overline{f(A)})$ [Since $f^{-1}(\overline{f(A)})$ is b-closed set in $\mathrm{E}$ and by remark ]. We have $f(\bar{A}) \subseteq$ $f^{-1}(\overline{f(A)})$.

ii. Let $f$ be sequentially bornological continuous. Since $\bar{B}$ is b-closed set in $\mathrm{F}$, then $f^{-1}(\bar{B})$ is b-closed in $E$ (theorem 2.7).

$\overline{f^{-1}(\bar{B})}=f^{-1}(\bar{B}) \ldots(1)$. Since $B \subset \bar{B}$ , then $f^{-1}(B) \subseteq f^{-1}(\bar{B})$. We have $\overline{f^{-1}(B)}=\overline{f^{-1}(\bar{B})}=f^{-1}(\bar{B})[$ by $(1)]$.

\subsection{Theorem:-}

Let $E, F$ and $H$ be bornological vector spaces and let $f: E \rightarrow F$ be a sequentially bornological continuous at a point $x, g: F \rightarrow H$ be a sequentially bornological continuous at $f(x)$ then $g \circ f: E \rightarrow H$ is be a sequentially bornological continuous at a point $x$.

\section{Proof}

Let $\left\{x_{n}\right\}$ be a sequence in $E$ such that
$x_{n} \stackrel{M}{\rightarrow} x$. since $f$ is a sequentially bornological continuous at $\mathrm{x}$ then $f\left(x_{n}\right) \rightarrow f(x)$.

Since $\left\{f\left(x_{n}\right)\right\}$ a sequence in $E$. Since $g$ is a sequentially bornological continuous at $f(x)$ then $g\left(f\left(x_{n}\right)\right) \rightarrow g(f(x))$. Then $(g \circ f)\left(x_{n}\right)$ $\rightarrow(g \circ f)(x)$. Hence $g \circ f$ is a sequentially bornological continuous at $x$.

\section{The Sequentially Bornological Compact Space}

In this section, we introduce the definition of bornivorous set and most important properties of it.

\subsection{Definition:-}

A subset $A$ of bornological vector space $E$ is called sequentially bornological compact if every sequence in $A$ has a convergent subsequence. And $E$ is called sequentially bornological compact space if every sequence in $E$ has a convergent subsequence .

\subsection{Theorem:-}

Every sequentially bornological compact in bornological vector space is complete.

\section{Proof}

Let $\left\{x_{n}\right\}$ be a Cauchy sequence in $E$. Since $E$ is sequentially bornological compact then by (definition 3.1) the sequence $\left\{x_{n}\right\}$ has a subsequence $\left\{x_{n_{k}}\right\}$ converging to $x \in X\left[x_{n_{k}}\right.$ $\stackrel{M}{\rightarrow} x]$. Then by (theorem 1.7) $\left\{x_{n}\right\}$ converges to $x_{0}$ hence $E$ is complete separated bornological vector space.

\subsection{Theorem:-}

Let $E$ be a sequentially bornological compact space. A subset $E$ of $E$ is b-closed if and only if it sequentially bornological compact.

Proof

Let $A \subseteq E$ be a b-closed. Let $\left\{x_{n}\right\}$ be a sequence in A. Let $\left\{x_{n}\right\}$ be a sequence in $E$. 
Since $E$ be a sequentially bornological compact space, then $\left\{x_{n}\right\}$ has a convergent subsequence $\left\{x_{n_{k}}\right\}$ such that $x_{n_{k}} \stackrel{M}{\rightarrow} x$.

Since $A$ be b-closed then by (definition 1.9) then $x \in A$ hence $\left\{x_{n}\right\}$ has a convergent subsequence in $A$, then $A$ is sequentially bornological compact.

\section{Conversely :-}

Let $A$ be sequentially bornological compact set. Let $\left\{x_{n}\right\}_{n \in N} \subseteq A$ and $x_{n} \stackrel{M}{\rightarrow} x$

Since $A$ is sequentially bornological compact set then $\left\{x_{n}\right\}$ has a convergent subsequence $\left\{x_{n_{k}}\right\}$ in $A$. Since $x_{n} \stackrel{M}{\rightarrow} x$ by (remark 1.4) we have $x_{n_{k}} \stackrel{M}{\rightarrow} x$ and $x \in A$. Hence $A$ is b-closed set.

\subsection{Theorem:-}

Let $f$ be a sequentially bornological continuous mapping of a sequentially bornological compact space $E$ into bornologicla vector space $F$. Then $f(E)$ is a sequentially bornological compact.

\section{Proof}

Let $f\left(\left\{x_{n}\right\}_{n \in N}\right)$ be a sequence in $f(E)$. Then $f\left(f\left(\left\{x_{n}\right\}_{n \in N}\right)\right)=\left\{x_{n}\right\}_{n \in N}$ is a sequence in $E$. Since $E$ is compact then $\left\{x_{n}\right\}$ has a convergent subsequence $\left\{x_{n_{k}}\right\}$ such that $x_{n_{k}} \stackrel{M}{\rightarrow} x$ in $E$. Since $f$ sequentially bonological continuous map then $f\left(x_{n_{k}}\right) \rightarrow f(x)$ hence every sequence in $f(E)$ has a convergent subsequence.

Then $f(E)$ is a sequentially bornological compact.

\subsection{Corollary:-}

Let $E$ and $F$ be bornological vector spaces and let $A$ be a sequentially bornological compact subset of $E$ if $f: E \rightarrow F$ is sequentially bornological continuous then $f(A)$ is a sequentially bornological compact subset of $F$.

\section{Proof}

The restriction $f_{A}: A \rightarrow F$ is sequentially bornological continuous mapping (theorem 2.6).

Since $A$ is a sequentially bornological compact of $\mathrm{E}$ then by (theorem 3.4) $f(A)$ is a sequentially bornological compact subset of $F$.

\subsection{Theorem:-}

Let $E$ and $F$ be sequentially bornological compact spaces if and only if $E \times F$ be sequentially bornological compact spaces.

\section{Proof}

Let $\left\{x_{n}\right\}_{n \in N}$ a sequence in a product bornological vector space $E \times F$ then $\left\{x_{n}\right\}_{n \in N}=\left\{x_{n}{ }^{1}\right\}_{n \in N} \times\left\{x_{n}{ }^{2}\right\}_{n \in N}$ such that $\left\{x_{n}{ }^{1}\right\}$ is a sequence in $\mathrm{E}$ and $\left\{x_{n}{ }^{2}\right\}$ is a sequence in $F$.

Since $E$ and $F$ are sequentially bornological compact spaces, then $\left\{x_{n}{ }^{1}\right\}$ has a convergence subsequence $\left\{x_{n_{l}}{ }^{1}\right\}$ in $\mathrm{E}$ and $\left\{x_{n}{ }^{2}\right\}$ has a convergence subsequence $\left\{x_{n_{m}}{ }^{2}\right\}$ in $F$ if $k=\min \{l, m\}$

$\left\{x_{n_{k}}\right\}=\left\{x_{n_{l}}{ }^{1}\right\} \times\left\{x_{n_{m}}{ }^{2}\right\}$ then by (proposition 1.5) convergence in $E \times F$ hence $E \times F$ be a sequentially bornological compact.

\section{Conversely:-}

Let $E \times F$ be sequentially bornological compact space

If $\left\{x_{n}{ }^{1}\right\}$ and $\left\{x_{n}{ }^{2}\right\}$ are sequences in $E$ and $F$ respectively, then $\left\{x_{n}\right\}_{n \in N}=\left\{x_{n}{ }^{1}\right\} \times$ $\left\{x_{n}{ }^{2}\right\}_{n \in N}$ be a sequence in $E \times F$. Since $E \times F$ is sequentially bornological compact, then $\left\{x_{n}\right\}_{n \in N}$ has a convergence subsequence $\left\{x_{n_{k}}\right\}=\left\{x_{n_{k}}{ }^{1}\right\} \times\left\{x_{n_{k}}{ }^{2}\right\}$ such that $\left\{x_{n_{k}}{ }^{1}\right\}$ convergence subsequence of $\left\{x_{n}{ }^{1}\right\}$ in $\mathrm{E}$ and $\left\{x_{n_{k}}{ }^{2}\right\}$ convergence subsequence of $\left\{x_{n}{ }^{2}\right\}$ in $\mathrm{F}$, hence $\mathrm{E}$ and $\mathrm{F}$ are sequentially bornological compact spaces.

\section{References}

[1] H. Hogbe Nlend, Bornologies and Functional Analysis, Netherlands: North -Holland Publishing Company Netherlands, 1977.

[2] S. a. D. P. Dierolf, "Bornological Space Of Null Sequences," Arch. Math., vol. 65, no. 1, pp. 46-52, 1995.

[3] O. S. V.I. Bogachev, Topological Vector Spaces and Their Applications, Springer Nature, 2017. 
[4] F. K. M. Al-Basri, "On Complete Convex no. 2, pp. 65-76, 2014.

Bornological Vector Spaces," Journal of $A L$-Qadisiyah for pure science, vol. 11, no. 1, pp. 139-147, 2014.

[5] A. N. D. Al-Salhi, "On Bornological Constructions," College of education journal, no. 1, pp. 131-141, 2007.

[6] F. K. M. Al-Basri, "The Relationship Between Boronological Convergence of Net and Topological Convergence of Net," Journal of AL-Qadisiyah for computer science and mathematics, vol. 6 ,

الفضاء البرنولوجي المرصوص تتابعيا

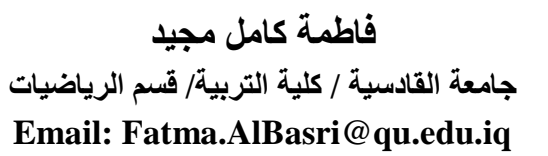

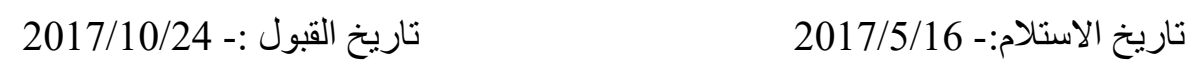

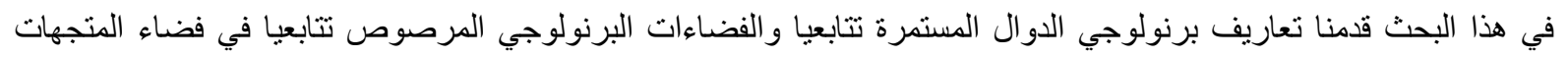

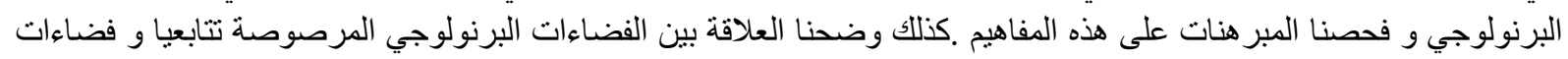

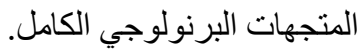

